\title{
Causal Analysis of Internet Public Opinion Dissemination System Based on the System Dynamics
}

\author{
Feng Cao ${ }^{1}$, Zhenji Zhang ${ }^{1}$, Erdan $\mathrm{Qi}^{1}$ and Xiaolan Guan ${ }^{2 *}$ \\ 1 Beijing Jiaotong University,Beijing 100044, China \\ 2 Beijing Institute of Graphic Communication, Beijing 102600, China \\ E-mail: \{09113108, zhjzhang, 12120608,08113101\}@bjtu.edu.cn
}

\begin{abstract}
Internet public opinion reflects the trend of public opinion in all sectors of society directly. It can affect the harmony and stability of society. Therefore reducing the incidence of adverse events and improving the ability to deal with Internet public opinion have become an important issue that the government needs to solve. Based on the existing literature, the paper firstly analyzes the propagation rule of Internet publicopinion and its features. Then it uses the system dynamics to have an analysis of the influencing factors of ecological dissemination system about internet public opinion. What's more, it takes a qualitative analysis to analyze the relationship between the actors through the causal analysis diagrams and it establishes the stock flow diagram of the factors. Lastly, ithas a simulation analysis for the model in order to present the suggestions to controthe dissemination of Internet public opinion positively.
\end{abstract}

Keywords: Internet public opinion, dissemination system, system dynamics, causal analysis

\section{Introduction}

With the rapid development of the Internet, the micro-blog, blog, BBS, news, email have been made into a diversified channel of communication for the public People's desire for selfexpression and di ersity of language and views have made the Internet "a free-market of views." As Juergen Habermas said, "Internet promotes the spread of egalitarianism, but we also have paid the price. The information for the decentralized readers is without filtering. Previous intellectuals are skill in communicating readers and information are increasingly lost their power of manafacuring focus in such a society."[8] Internet public opinion reflects the trend of public opinion in all levels of society fast and more directly. It has become a realtime social barometer of public opinion. So the government attaches great importance on it. On the internet, a series of deep social contradictions and events about people' s sensitive, vulnerable psychosocial problems have caused widespread concern. If the guide is undeserved, it may lead some users to lose their reason, which will have a huge destruction. Therefore reducing or even eliminating the occurrence of adverse events and improving the ability to deal with Internet public opinion and to maintain stability of the society have become an important issue for the government.

If the government can not properly grasp the law of Internet public opinion to guide its dissemination, it will have an adverse impact on the economic development and social harmony. So the research on the Internet public opinion has become a hot research. In the early eighteenth century, the French Rousseau first proposed the concept of "public opinion". From the twentieth century, the concept of public opinion began to be widely used in many 
countries, promoting the development of the social public opinion surveys and the test of public opinion [13].Liu Yi believes that Internet public opinion is a staggered sum of a variety of emotions, attitudes and opinions from a variety of social groups .The social groups take care for the public office that they are interested in, or closely associated with in a certain social space [10].Ji Hong and Ma Xiaojie believe the Internet public opinion refers to the netizens' the attitude to those in power and the users' political orientation about the public opinion can be changed due to the occurrence, development and change of the events [11].In the aspect of formation and development of the Internet public opinion, Zhang Yong feng pointed out that the views expressed on the internet have its source in the phenomenon and problems that exist in real life through analyzing the information about "Travel News" in the "quantity" and "quality" [1]. Zhou ru Jun and Wang Tian qi think that the Internet public opinion are formed mainly from the social conflicts, extensions and infrequent event [9], In the aspect of guidance and control, Wong KamC analyzed the impact on the public behavior and the path by discussing the relationship between public opinion, national policies and the media[2]. Xu Xiaolin and Wang Ziwen believe that it should take the initiative to grasp the situation and have a response in a positive and scientific way so that the government can grasp the right of guidance about the internet public opinion firmly [12]. In terms of treatment method, Liu Yi studied the role of content analysis in the internet public opinion [3]. Huang Xiaobin studied the role of text data mining method about the Mntenet public opinion [4]. When analyzing the impacts to journalists, Brooks, B. believed that, in the Internet communication, the role of journalists as gatekeepers will be reduced and that the existing media agenda setting in the network environment is still discussed [14]. Bart W. Edes had an analysis of the government information officer (GIO). He believed that the role of spokesman is significant in helping the public understand the government policy. What' s more, it can improve the public ability to know the policy and promote communication between the public and the media [15].

However, there are not many researches of dynamics about the propagation process of Internet public opinion. In the paper, based on the previous literatures, it analyzes the factors that affect the spreâd of the ecological Internet public opinion. And it provides some recommendations to ensure the effective dissemination of the network of public opinion.

\section{Characteristics of the ecological propagation about Internet public opinion}

Information dissembation of the Internet public opinion is divided into three stages: The first stage is that the major authority sites and other means give much coverage. And traditional medias' reports on the website have a reinforcement effect to increase the effect of the site.

The second stage is the forwarding phase, but also a key step of the communication. It relates to the scope and degree of the Internet public opinion dissemination. And the reproduced information channels include the forum, each big website reprint and so on.

The third stage is that the users have incentive discussions in various forums or chat room, forming different views and opinions, and even leading to conflict, so as to form the public opinion hot spot.

Network transmission has the following characteristics: 


\subsection{Freedom}

Freedom embodied in that the Internet users can place the "facts" in the "public domain" network to make it easy to be believed the "facts" through the Email, IM, BBS, blog, micro-blog and so on many kinds of ways. Internet users can transmit the information and express the public opinion more free. Internet users can also establish their own web site, or "publish" their own books or newspapers to express their opinions.

\subsection{Controllability}

Generally speaking, every new media will further expand the degree of freedom of people's spreading the news. This is the general rule of communication technology development history. Controllability refers to that it can control and restrict the propagation of Internet public opinion by making the related laws and regulations.

\subsection{Instantaneity}

When the major emergencies occur, the network media can publish original news commentary in the first time and the users can arso express their views online in the first time.

\subsection{Far-reaching influence}

Network media can track to report the major incidents or issues and publish the information all the time. This not only expands the impact of the public opinion on the public, but also greatly enhancesthe effect which the public make on the public opinion events.

\section{Analysis of the driving force about the Internet public opinion dissemination system}

This paper has an analysis of the factors influencing the spread of the Internet public opinion from four aspects, the events, the internet, the media and the government.

\subsection{The force of the event}

The event of public opinion generated the Internet public opinion. Internet users interacting on the internet, the media coveraging the event and the government dealing with events can influence the change of the Internet public opinion.

3.1. The influence of events: As the main body of the event, people's social awareness and social influence can cause great influence on the effects of the events. The higher the social awareness is or the greater the social influence is, the greater the effect of the event is. When the event itself and the users' interests have a relative high correlation, it will also affect the effect of event.

3.1.2. The harm degree of events: The events of the Internet public opinion will bring harm to the people and the society, which make people feel discomfort and fear in the psychology. 
It will not only endanger social stability, but also affects the credibility of the government. The greater the harm is, the more fears the public feel and the hotter the Internet public opinions are. The action ratio of the event is the comprehensive of the effect of events and the harm degree of events. As long as any one of these factors plays a role, the Internet public opinion would be stimulated. The increasing of the action ratio will lead to more attention of the public, making the heat of the Internet public opinion high.

\subsection{The force of netizen}

Among the existing researches, most scholars believe that the Internet users are the subject of Internet public opinion. In the process of the formation of Internet public opinion, Internet users are not only the receiver of information, but also the transmitter of information. They obtain information from the network and express their views and exchange the opinions, which cause the government and relevant departments to pay more attention to the event, promoting the change and development of the public opinion.

3.2.1. The frequency of discussion: For the topic that people are concerned about, when the users search the information on the internet, if they discontent with the development trend of the event, they will vent their emotions through micro-blog, blog, forum, QQ and so on in order to find the consensus of the others. At the same time, they alsa transmit the information of the Internet public opinion to others face to face.

As the discussing frequency about the events of the Internet public opinion increased, people imitate and continue to affect each other, which promote the dissemination of the public opinion.

3.2.2. The role of opinion leaders: Opinion leaders are those who contact with the mass media frequently and tend to have deep and long-term views of events. They have a certain influence on the Internet users and can influence the behavior of others within a certain range. The role of opinion leaders is to spreat the information and ideas on the network and to guide the thinkings and actions of other Intermet users, making the Internet public opinion develop towards a certain direction louder.

When users views and Opinions of the leaders are similar, netizen will have a strong sense of identity and the public opinions will develop towards the initial direction more extremely, causing the Interhet public opinion rising. On the contrary, if the opinion leaders have the correct orientation, it will produce the guiding effect on the thought of Internet users .Then it can ease the Internet users " tensions.

Therefore, opinion leaders play an important role in the development and regression of public opinion on the network. Also, the government should do a good job of the opinion leaders so that they can play an active role in guiding public opinion' $\mathrm{s}$ trend and soothe the users' enotions.

3.2.3. The intensification of the internet groups: When some extreme views prevail, it is easy to form "groups intensified". It can result in some extreme behaviors which will serious affect the social harmony. The phenomenon of the Internet groups intensified is actually due to people' powerless to justice and self-preservation. They expected to reach a consensus with others to share responsibility and solve the problem. The mood is not only from the network, but also from the discussion of the people in real life. The high intensification 
degree of the Internet users will increase the public discussion largely, which would cause the heat of the Internet public opinion.

\subsection{The force of traditional media}

Media is the carrier of public opinion. It includes the internet and traditional media. Network media includes the major news sites such as Sina, Sohu and other important channel. Traditional media has three media, including television, newspapers and radio. In the information age, it can not ignore the impact of the traditional media while researching the Internet public opinion. The influence of networks has made the events to be the focus of the traditional media. Thus many traditional medias coverage it as a hot topic, lead the public to take more attention to the issue and then spread it on the internet. In that way, it deepens the mutual action between public opinion and real opinion.

3.3.1. The frequency of the media coverage: If the people do not have an accurate and satisfactory understanding of the event, it will increase the frequency of discussions online and offline, thus leading to the intensification of the internet groups and increasing the heat of the Internet public opinion. Media should have a real and timely coverage on the events to dispel the misunderstanding of the public. So that people can rationally understand the dynamics of the event and ease their negative enotions in a timely manner. So it can reduce the heat of the Internet public opinion.

3.3.2. The action rate of the media: The public have a great dependence on the key media. Media of greater visibility will have a great impact on the internet users. The higher the action rate of the media is, the greater the frequency of the media's coverage is .And it is easier to improve the heat of the Internetpublic opinion

\subsection{The force of the relevant government departments}

The role of the government in the development of the Internet public opinion is consistent .It is the key factor and decisive factor in recession of the Internet public opinion. Only the relevant government departments deal with the network public events and resolve the crisis of the Internet public opinion correctly and timely, can it guide the development of Internet public opinion rightly.

3.4.1. The attention of the government: Government is concerned about the people's livelihood and the society. When the event of the Internet public opinion occurs, the government will pay more attention to it .Then efforts of government crisis management and the efforts of processing the public opinion also increase, which will benefit to solve the crisis of Internet public opinion and to promote the reduction of heat. The high action ratio of the event will promote the government to pay more attention to the event.

3.4.2. The efforts of the government crisis management: The government should deal with the event effectively and timely, since the efforts of the government crisis management will affect the public's attitudes towards the national regulators and the credibility of the government. The efforts of the government crisis management include the satisfaction of dealing with the event and the response speed of the government. 
The satisfaction of dealing with the event is the satisfaction of the people on the government's crisis management. If the satisfaction is high, the heat of the Internet public opinion will be reduced quickly. If the government failed to deal with it timely and effectively, the event would not be resolved fundamentally and the situation would develop in the direction towards the vicious heat of public opinion. It will make the heat of the Internet public opinion increase, constituting a crisis of the Internet public opinion even secondary events. In this case, netizens will doubt the government working ability, management ability and the credibility, which will deepen the emotional instability of the Internet users.

The response speed of the government refers to the speed that the relevant government departments make corresponding response to the events. It will not only reduce the harm and loss that the events bring to the people and society, but also reflect the government's work attitudes. If the government response to the event fast and treat it timely, it will reduce the harm, which is conducive to the government's credibility. So that the heat of the Internet public opinion can be reduced.

3.4.3. The government' $s$ efforts to deal with the public opinion: The government' $s$ efforts to deal with the public opinion is that in the early time, the governments make responses to the reaction of the public and media in order to guide the thinkings and behaviors of the internet users. Thus it can control the development of the Internet public opinion. The government' s efforts to deal with the public opinion include the degree of information disclosure, the authority of the spokesman; and the role of opmion leaders.

The governments will publish the information of the events and the development of measures and actions taken by the government through the official website or media timely and transparently. So the process of the event can betransparent .In this way, the public can understand the development and the processes of events. Thus the government can guide people's thinkings into the right direction.

Credibility is the objectire result of the government's administrative capacity. It reflects the authority of the government and the degree of democracy. Meanwhile, it is also the masses' evaluation to the government and reflects the satisfaction and trust of the public to the government.

\section{Model}

\subsection{The diagram of causal analysis}

Causal relation is one of the basic tools of system dynamics. The diagram of causal analysis is a qualitative description of the graphical model describing the causal relationship between ariables in the system. This is based on the social system. System dynamics is the combination of system science and computer simulation, devoting to the study of system feedback structure and behavior science. It uses the qualitative and quantitative methods to do the simulation analysis and makes the research in a long-term, dynamic, strategic way [5]. There are two methods of analysis: diagram of causal analysis and flow chart.

In the diagram of causal analysis, variables are linked by causal chains. The chain of causation is represented by arrows and each causal chain is polar. Polar represents that when the variables change, the related variable change. When $\mathrm{A} \nearrow$ causes $\mathrm{B} \nearrow$, or $\mathrm{A} \searrow$ causes $\mathrm{B}$ $\searrow$, it indicates that the chain between $\mathrm{A}$ and $\mathrm{B}$ is a positive causal chain. When $\mathrm{A} \nearrow$ causes $\mathrm{B} \searrow$, or $\mathrm{A} \searrow$ causes $\mathrm{B} \nearrow$, it indicates a negative causal chain between $\mathrm{A}$ and $\mathrm{B}$. The causal 
loop diagram of factor analysis of the Internet public opinion can be built after analyzing the factors.

\subsubsection{Causality diagram of the government' $s$ forces is as figure1:}

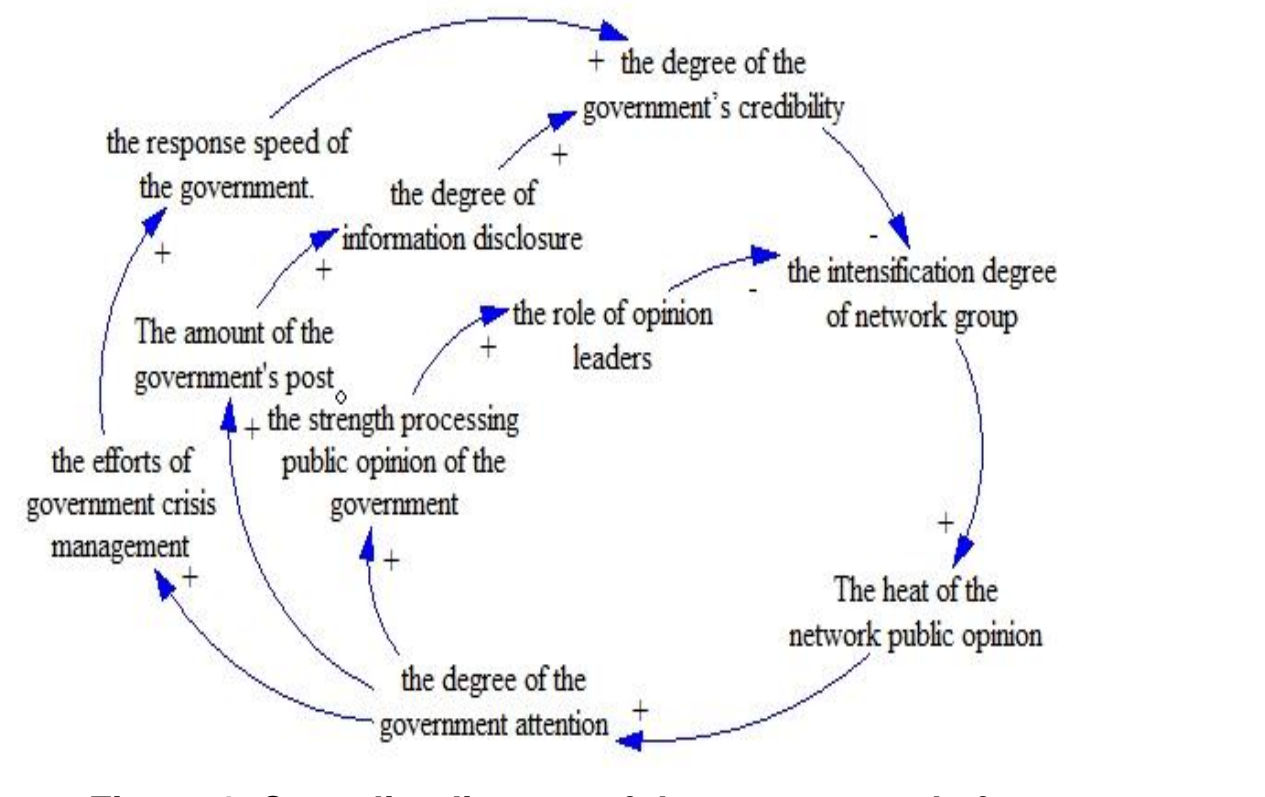

Figure 1. Causality diagram of the government's forces

4.1.2. Causality diagram of the netizen' forces is as figure2:

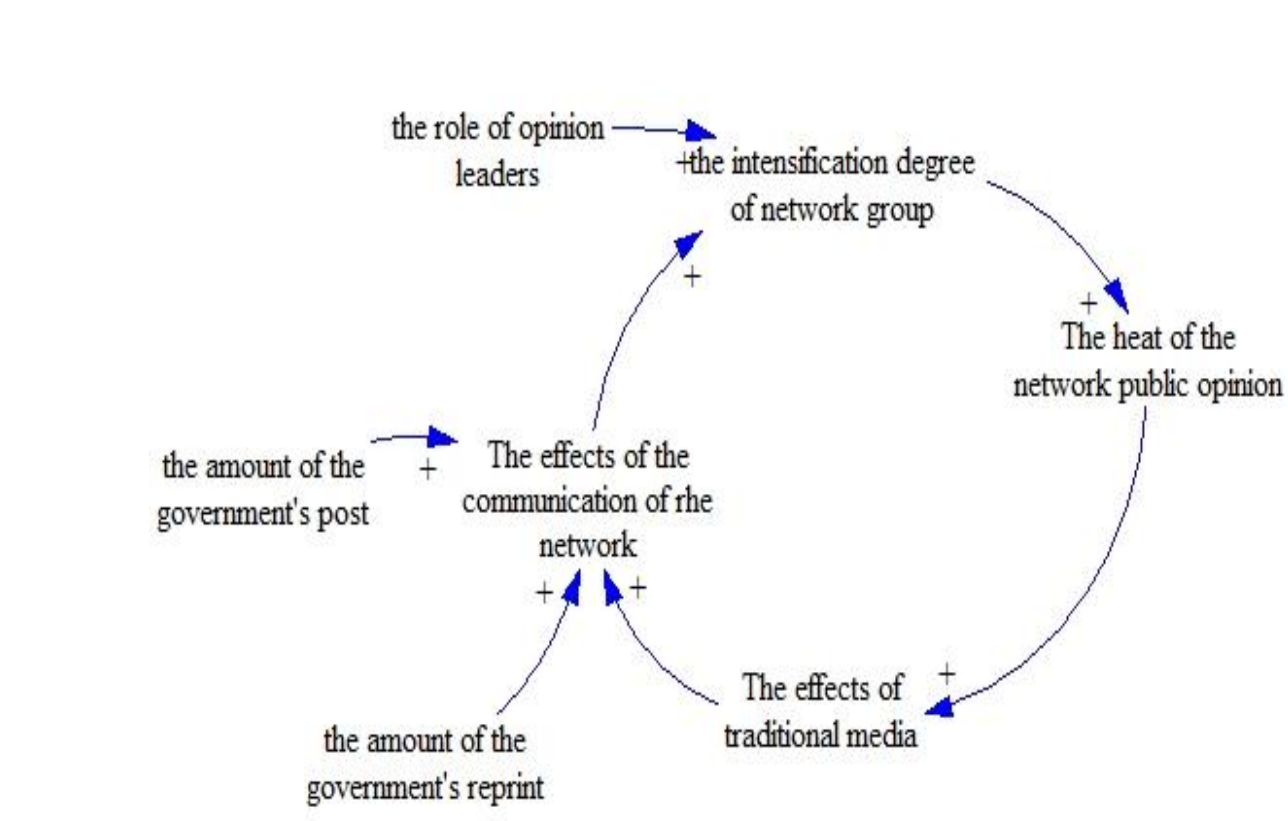

Figure 2. Causality diagram of the netizen's forces 


\subsubsection{Causality diagram of the traditional medias' forces is as figure3:}

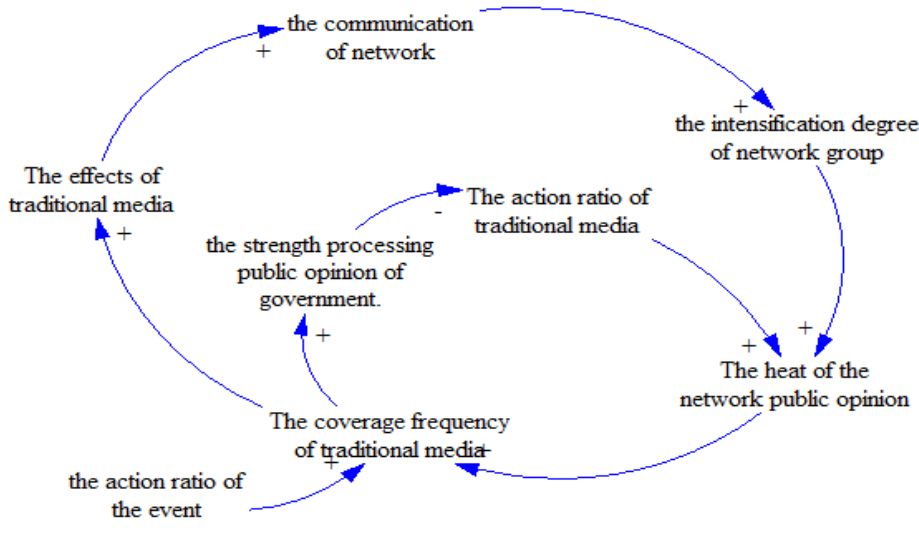

Figure 3. Causality diagram of traditional medias' forces

\subsubsection{Causal analysis and feedback diagram of the dissemination system of the Internet} public opinion is as figure4:

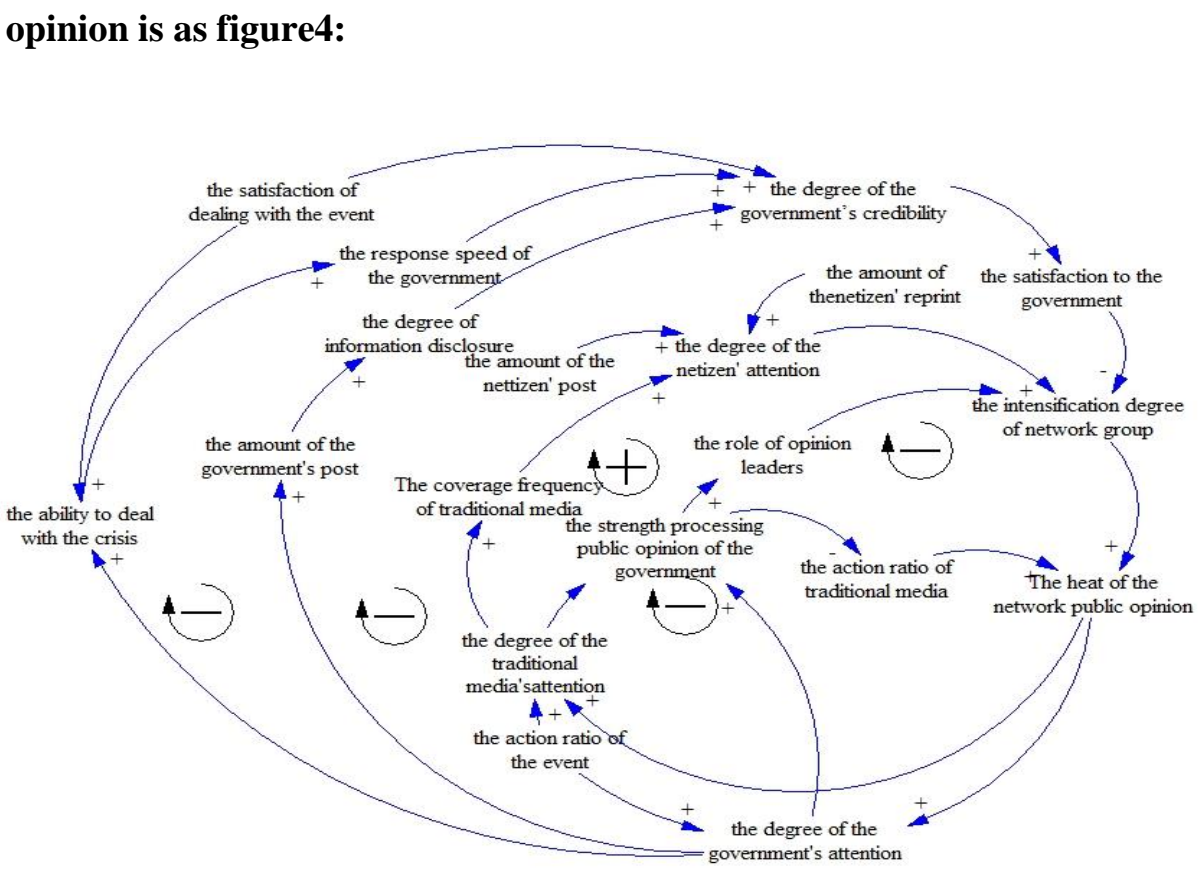

Figure 4. Causal loop diagram of factors for the dissemination system of public opinion

\subsection{Stock flow diagram}

Causal loop diagram only analyze the impact of various factors on the overall system loop, but it doesn' thave an analysis of the process of accumulation amount. Stock flow diagram is based on causal loop diagram to identify the specific stocks and flows. Flow refers to the 
change of the variable within a certain period of time, while the stock is the final amount of accumulation at a certain point.

Then, it uses the mathematical equations to show the logical relationship between the factors and it has a quantitative analysis by using a specific number of data mining to describe the dynamic process of complex events from the view of the system. It identifies the key indicators of each question on the basis of a causal loop diagram .There are three interrelated sub-loop systems of stock flow diagram for each question.

Stock flow diagram of the Internet public opinion dissemination system can be established as figure5:

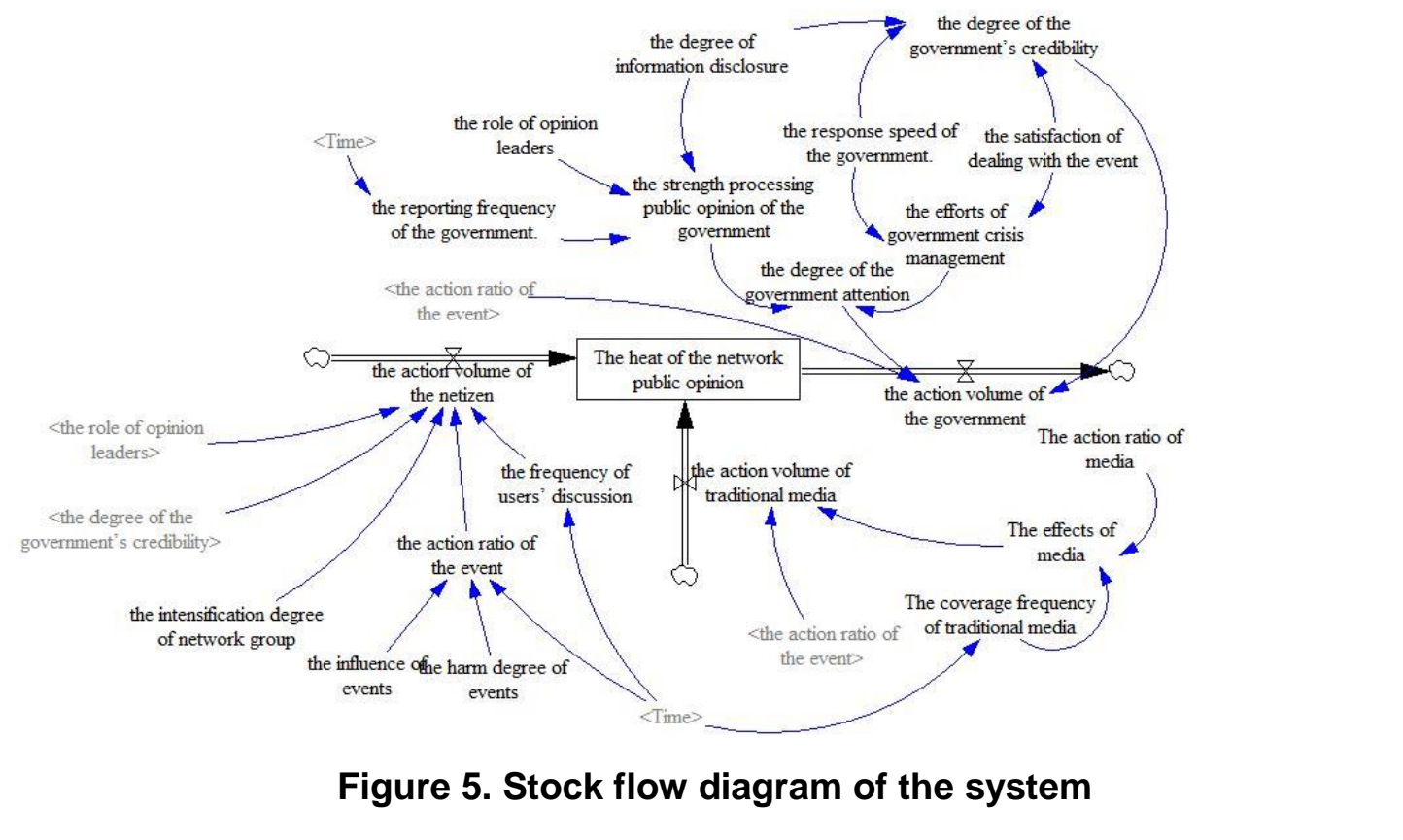

\section{Simulation analysis}

At 5:30 on August 24, 2013, Harbin Yang ming Beach Bridge opened less than a year was broken, resulting in four harge trucks falling bridge, three people killed and five people injured .

After the accident, Harbin quickly started the emergency response plans and set up the department of commanding about rescuing, investigating and dealing with the scene of the accident which is commanded by the municipal competent leadership.

The event has gone through a complete stage of occurrence, development, processing and result. The Internet public opinion also has the stage of generating, climax and regression.

Before the simulation, it needs to determine the various factors, including the constant parameters, table functions and the delay function.

\subsection{The function of the Variables}

(1) the action ratio of the event $=(0.58 *$ the harm degree of events $+0.42 *$ the influence of events) * EXP (-time)

Due to the occurrence of events and the development of the networks public opinion, it will be concerned by the public in the initial state, but will slowly fade over time and the public will be attracted by other events. Therefore, the action ratio of the event shows an 
index decrease over time in the action of the harm degree of events and the influence of events. The weights of the harm degree and the influence of events are scored by experts.

(2) The effects of media $=$ The action ratio of media * The coverage frequency of traditional media

It can use the table function in the dynamics software to represent the coverage frequency of traditional media by collecting and analyzing the news reports data on people.com.cn. It can get the action ratio of media by getting the questionnaire of 80 users and analyzing the data.

(3) the action volume of traditional media $=$ the action ratio of the event $*$ The effects of media

(4) the efforts of government crisis management $=$ the satisfaction of dealing with the event + the response speed of the government.

(5) the strength processing public opinion of the government $=$ (the degree of information disclosure+ the role of opinion leaders) * the reporting frequency of the goverment.

It can use the table function in the dynamics software to represent the reporting frequency of the government by collecting and analyzing the news reports data on websites of the government.

(6) the degree of the government attention= the efforts of government crisis management + the strength processing public opinion of government

(7) the degree of the government' s credibility= (the satisfaction of dealing with the event + the response speed of the government)* the degree of information disclosure/3

(8) the action volume of the government $=$ DELAYH (the action ratio of the event*( the degree of the government attention + the degree of the government' $\mathrm{s}$ credibility),1,0)

The action volume of the government can reduce the heat of Internet public opinion, but there is a certain delay. Only it delays one unit, namely one day, the action of the government can influence the heat of the publid opinion. The degree of information disclosure is obtained by getting the questionnaire of 80 users anc analyzing the data.

(9) the action volume ol the netizen = the intensification degree of network group*the frequency of users' discussion* the role of opinion leaders* the action ratio of the event/ the degree of the government' s credibility

It can use the table function in the dynamics software to represent the frequency of users' discussion by collecting and analyzing the news reports data on Tianya Forum.

(10) The heat of the Internet public opinion=INTEG $(0.164 *$ the action volume of traditional media+ $0.583 *$ the action volume of the netizen- $0.253 *$ the action volume of the government, 0)

The heat of the Internet public opinion is influenced by three factors directly. The weights of them are bbtained by the expert scoring through AHP and the final results use the average weight, while the initial value is set to 0 .

(11) In the model, the influence of events, the harm degree of events, the intensification degree of network group, the role of opinion leaders, the action ratio of media, the satisfaction of dealing with the event, the degree of information disclosure and the response speed of the government are the constant parameters. According to the expert scoring method, questionnaire method, weighted average method and the arithmetic average method, their values are $90.25,83.25,77.25,46.5,66.36,18.96,30.65,36.86$. 


\subsection{The table functions}

In the formation of the Internet public opinion crisis, the attentions of big forums, news sites, traditional medias and network medias embodied in the frequency of users' discussion, the reporting frequency media and the coverage frequency of the government. As to "Harbin in the yangming bridge collapse", this paper selects Tianya, people.com.cn, sina.com.cn, people's government website of Heilongjiang province as data source to collect data .It uses the table function to show the reporting number of the events of each Internet public opinion dissemination channel, which represent the frequency of the reporting.

(1) The frequency of users' discussion =WITH LOOKUP (Time, $([(0,0)-(10,1500)]$, $(0,236),(1,1496),(2,856),(3,468),(4,291),(5,68),(6,201),(7,80),(8,91),(9,25),(10$, $15))$ ), the simulation diagram can be obtained by using Vensim-PLE software. It can be seen as figure 6:

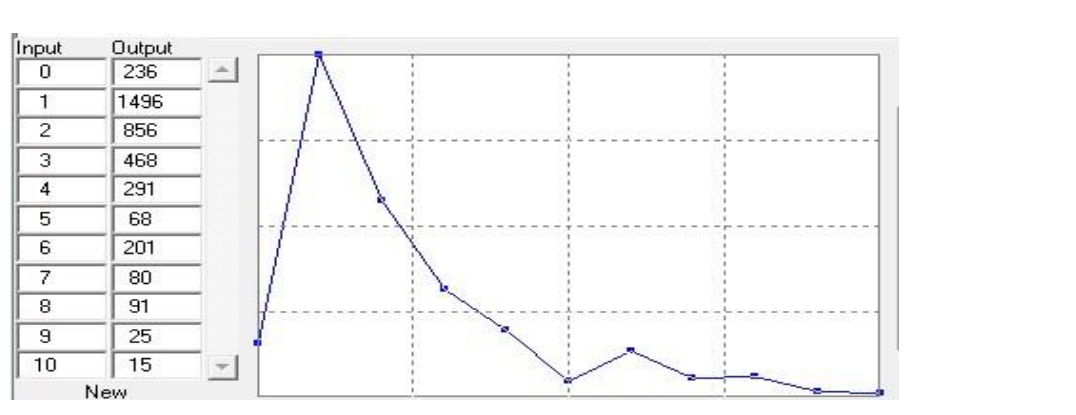

Figure 6. The table function of the frequehcy of users' discussion

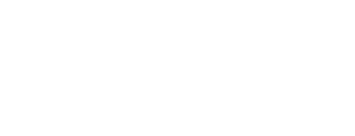

(2) The coverage frequercy of the goyernment $=$ WITH LOOKUP (Time, $([(0,0)-(10,80)$, $(0,2),(1,10),(2,78),(3,65),(4,36),(5,18),(6,34),(7,40),(8,19),(9,23),(10,5)))$, the simulation diagram can be obtained by using Vensim-PLE software. It can be seen as figure 7:
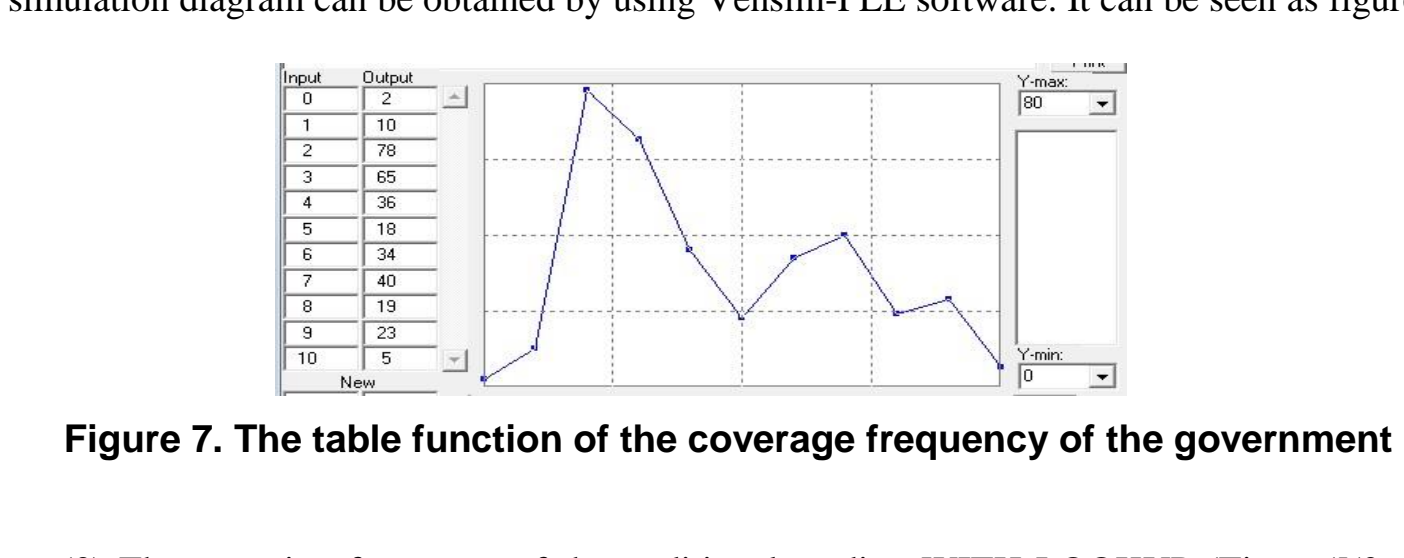

(3) The reporting frequency of the traditional media $=$ WITH LOOKUP (Time, $([(0,0)$ $(10,100),(0,12),(1,89),(2100),(3,57),(4,73),(5,69),(6,20),(7,0),(8,6),(9,3),(10,6)))$, the simulation diagram can be obtained by using Vensim-PLE software.It can be seen as figure 8: 


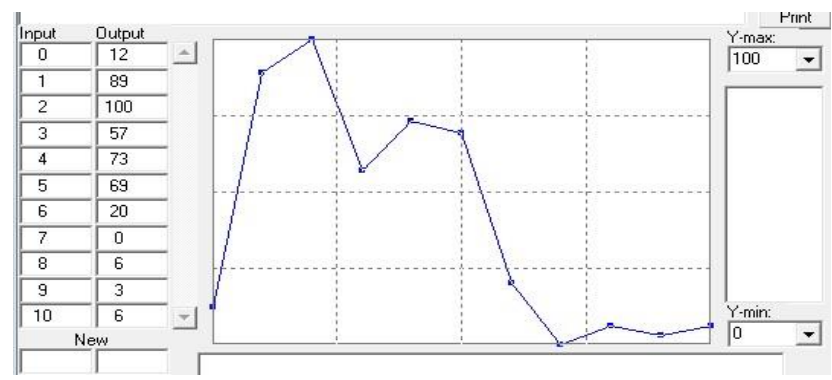

Figure 8. The table function of the reporting frequency of the traditional media

\subsection{Simulation results and analysis}

By running the results we can get the relationship between the heat of the Internet public opinion and three factors: government, users and the traditional media and the relationship between these factors with time. It can be seen in the figure 9 .

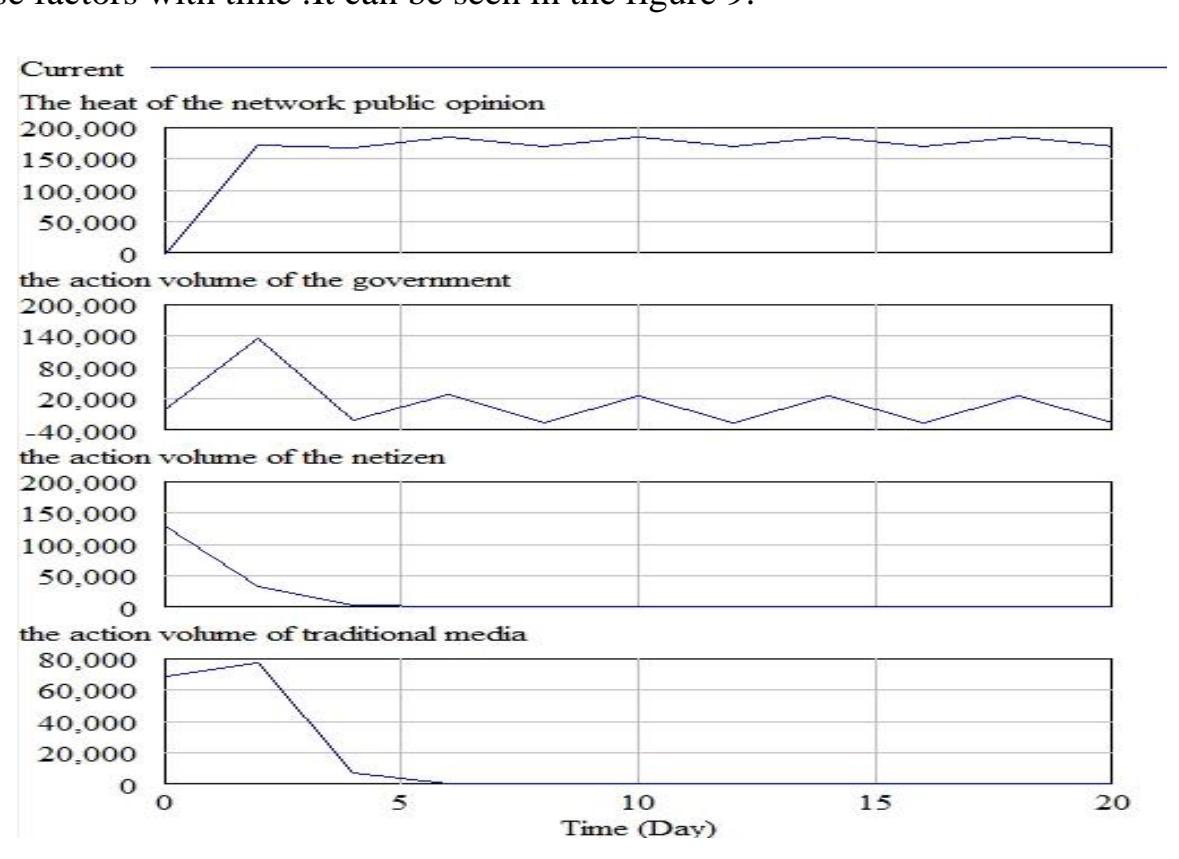

Figure 9. The contrast of results

5.3.1. The subsystem of government: In the government subsystem, the satisfaction of dealing with the event, the degree of information disclosure and the response speed of the government jointly make effects on the heat of the public opinion, affecting the credibility of the go ernment. When these factors are increased by $30 \%$, the heat of the public opinion has a certain of reduction. It can be seen in the figure 10 . 


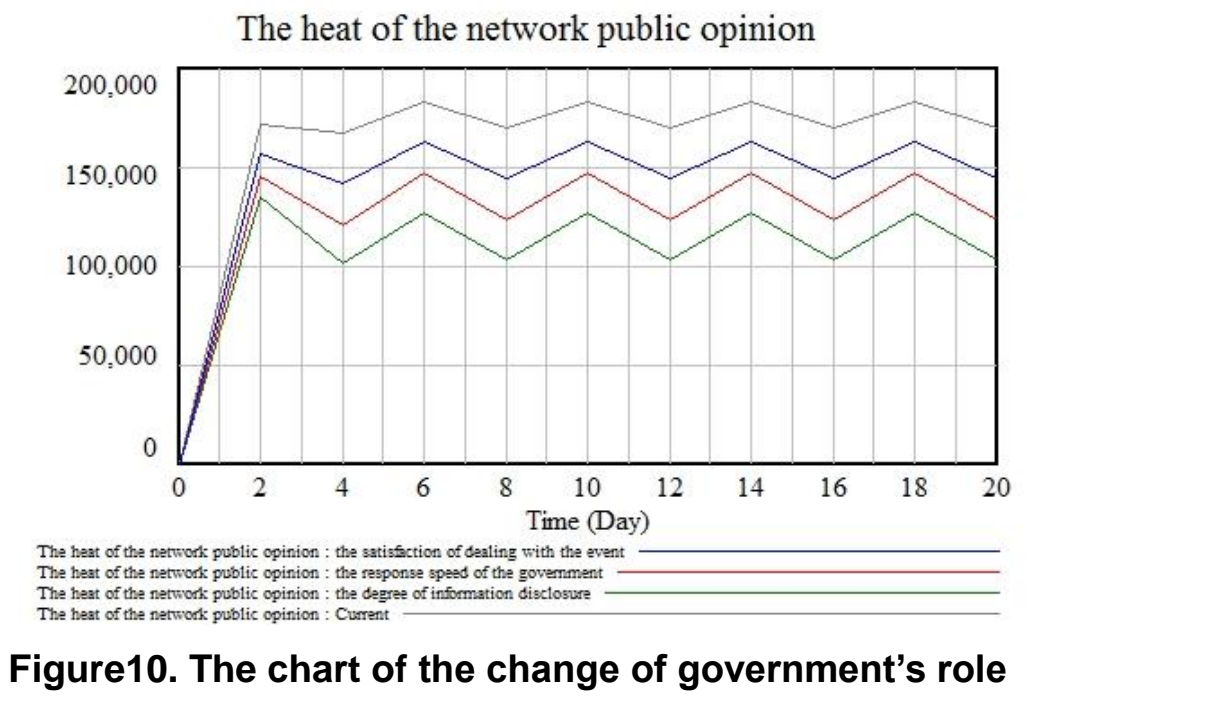

5.3.2. The subsystem of netizen: In this system, the role of opinion leaders and the intensification degree of network group have the important influence on the Internet public opinion. If we let the influence of the opinion leaders increase by $30 \%$ and reduce by $30 \%$, it can be seen that increasing the role of opinion leaders will leadt the increasing of the heat of the public opinion, Otherwise decreasing. When the intensification degree of network group has a reduction of $30 \%$, we can see the heat of the publie opinion is also reduced. It can be seen in the figure 11.

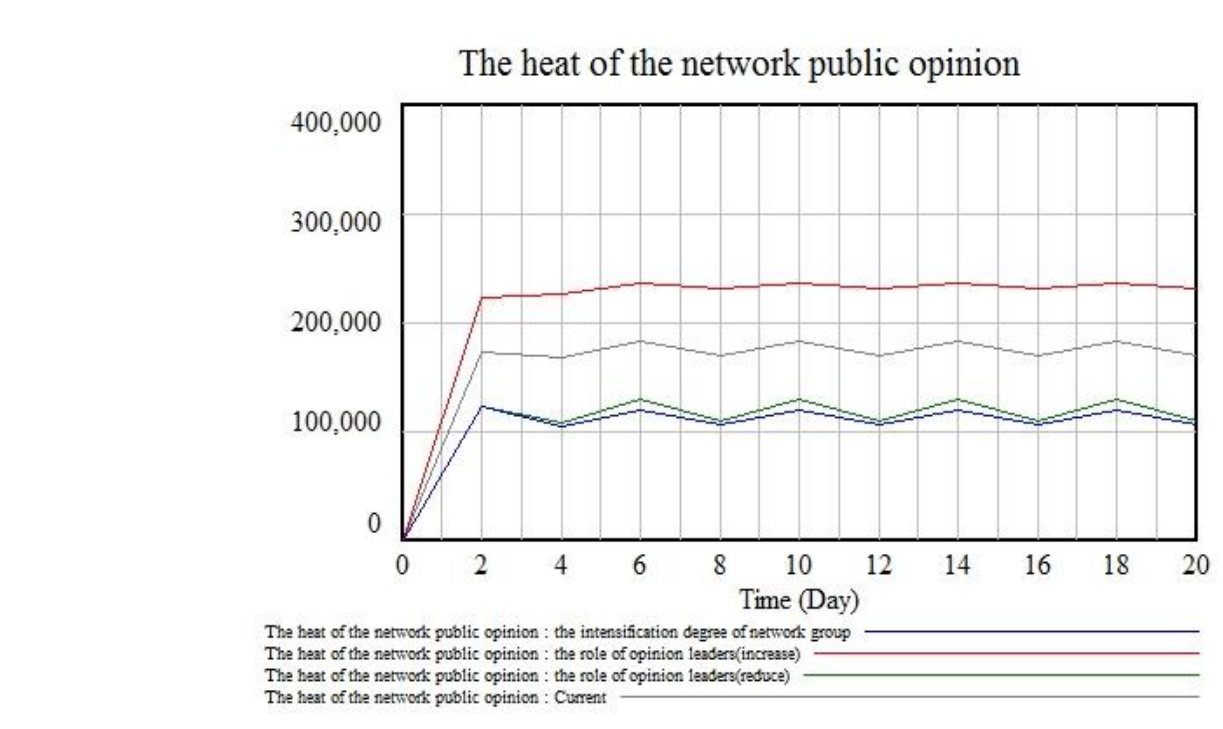

Figure 11. The chart of the change of netizen's role

5.3.3. The subsystem of events' force: In the subsystem, the harm degree of events and the influence of events affect the development of the heat of the Internet public opinion. If these two factors were reduced by $30 \%$, we can see the heat of the public opinion also be reduced. It can be seen in the figure 12 . 


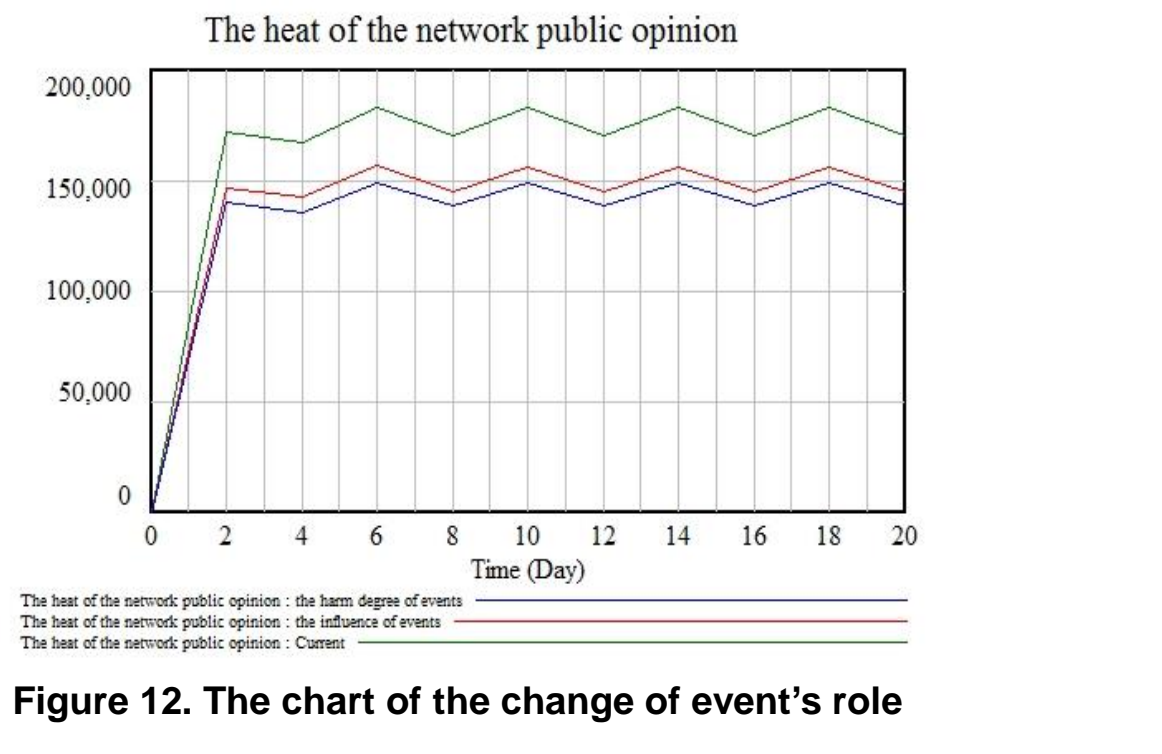

5.3.4. The subsystem of the traditional media: As you can see, the action ratio of media has an important influence on the heat of the public opinion. When the lactor is decreased by $30 \%$, the heat of the public opinion also be reduced. It can be seen in the figure 13 .

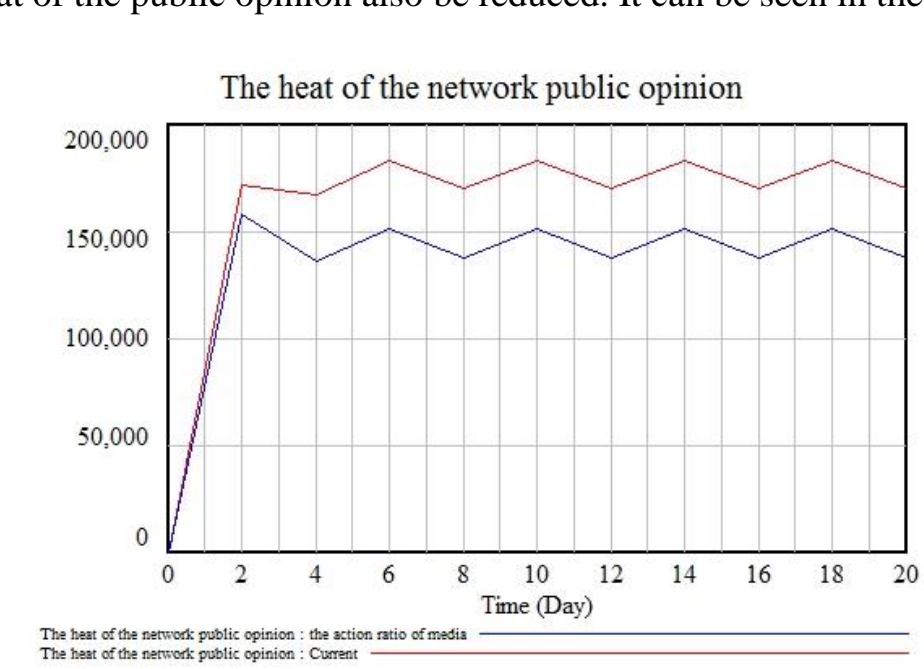

\section{Figure 13. The chart of the change of traditional media's role}

\section{Conctusion}

Through analyzing the causality diagram, we can see that the traditional way of the government responding to the Internet public opinion has not been adapted to the new media environment. The working mechanism dealing with dissemination of the Internet public opinion is not perfect and it lacks the communication ability. At the same time, the comprehensive coordination ability of third party and the ability of eliminating crisis have a scarcity. The government needs to collect all kinds of information in first time and formulate the corresponding measures to guide the dissemination of the Internet public opinion timely and effectively. 


\subsection{Enhance the management level of the government organization system about the Internet public opinion}

The government should pay attention to the management of the spreading of the Internet public opinion. It can improve the construction of administrator organizations in order to improve the ability of managing information on the internet. What' s more, the government should enhance the management and guidance level of the Internet public opinion. What's important, it should publish and supervise the daily information on the internet effectively so that it can discover and guide the Internet public opinion timely.

\subsection{Strengthen the cooperation between the government and the media}

The role of the media is important to the heat of the Internet public opinion. The government and the media should improve the transparency of the information. So people can understand the truth. It can reduce the degree of the Internet opinion emergencies and the degree of the harm.

In the early dissemination of the public opinion the grass-roots organs report the related information about the event timely and then the government starts the monitor of the public opinion in the first time. Thus the government can reach on countermeasures and prepare the responding plan to publish the comprehensive, accurate, authoritative information througl T) and the government website. Then it can block the channel of spreading the rumors to enhance the credibility of the government. According to the type of events and he situation, it can have a positive guidance of the Internet public opinion and notify the development of the event to the Internet users through the media.

\subsection{Pay attention to the "Opinion leaders"}

The government should also seatch for the "opinion leaders" online, take care for their speeches and strengthen the communication with them in order to guide them to understand the policy of the government and encourage them to provide more constructive opinions.

\section{Acknowledgements}

This work was supported by a grant from the Major Program of Beijing Planning office of Philosophy and Social Science (No. 13ZDB10).

\section{Reference}

[1] Yong feng, Zhang, J.The today media.The analysis of network public opinion On "Tourism News" event. 9,44 (2008)

[2] Wong Kam C, J. International Journal of the Sociology of Law.The making of the USA PATRIOT Act II : Public sentiments, political gamesmanship, media patriotism. 34,3 (2006)

[3] Yi,Liu, J. Theorists. The description On the concept features, expression and dissemination of network public opinion. 5,1 (2005)

[4] Xiaobin,Huang,Chao,Zhao,J. Information Science.Text mining applications in the network analysis of public opinion information . 27,1 (2009)

[5] Yongguang,Zhong,System dynamics, The Science Press, Beijing(2009) 
[6] Jingtao,Zhang. J. The China - ASEAN Expo.Discussion on the guidance of enterprise about the network public opinion. 11,6 (2011)

[7] Yiwen,Zhang,Jiayin, J. The Information reaserch. Qi. Internet public opinion and unconventional emergency mechanism - based on system dynamics modeling.29,9 (2010)

[8] [U.S.] Andrew - Keane. Internet users Carnival: Reflections on the Internet drawbacks . Dingde Liang translation. Nanhai Publishing Company, Haikou (2010)

[9] Ru Jun,Zhou, Tianqi,Wang,J. Ideological and theoretical education. network public opinion: the new areas of modern ideological and political education . 7,11 (2005)

[10] Yi Liu.J. Frontier.Changes of network public opinion and governance paradigm. 14,10 (2006)

[11] HongJi, Xiaojie,J. Huazhong University of Science and Technology (Social Science Edition).Ma. the collection, analysis and guidance of network public opinion.11,6 (2007)

[12] Xiaolin,Xu, Ziwen ,Wang.J. Management World .The research of issue about network public opinion.11,4 (2010)

[13] Wenbo,Kuang. Introduction to Network Communication. Higher Education Press, Beijing (2001)

[14] Brooks,B.Journalism in the information Age-A Guide to Computers for Reporters and Eclitors .Allyn and Bacom, 1997.

[15] BartW.Edes.J.Journal of Government Information."The role of government information officers". $23,48(2000)$

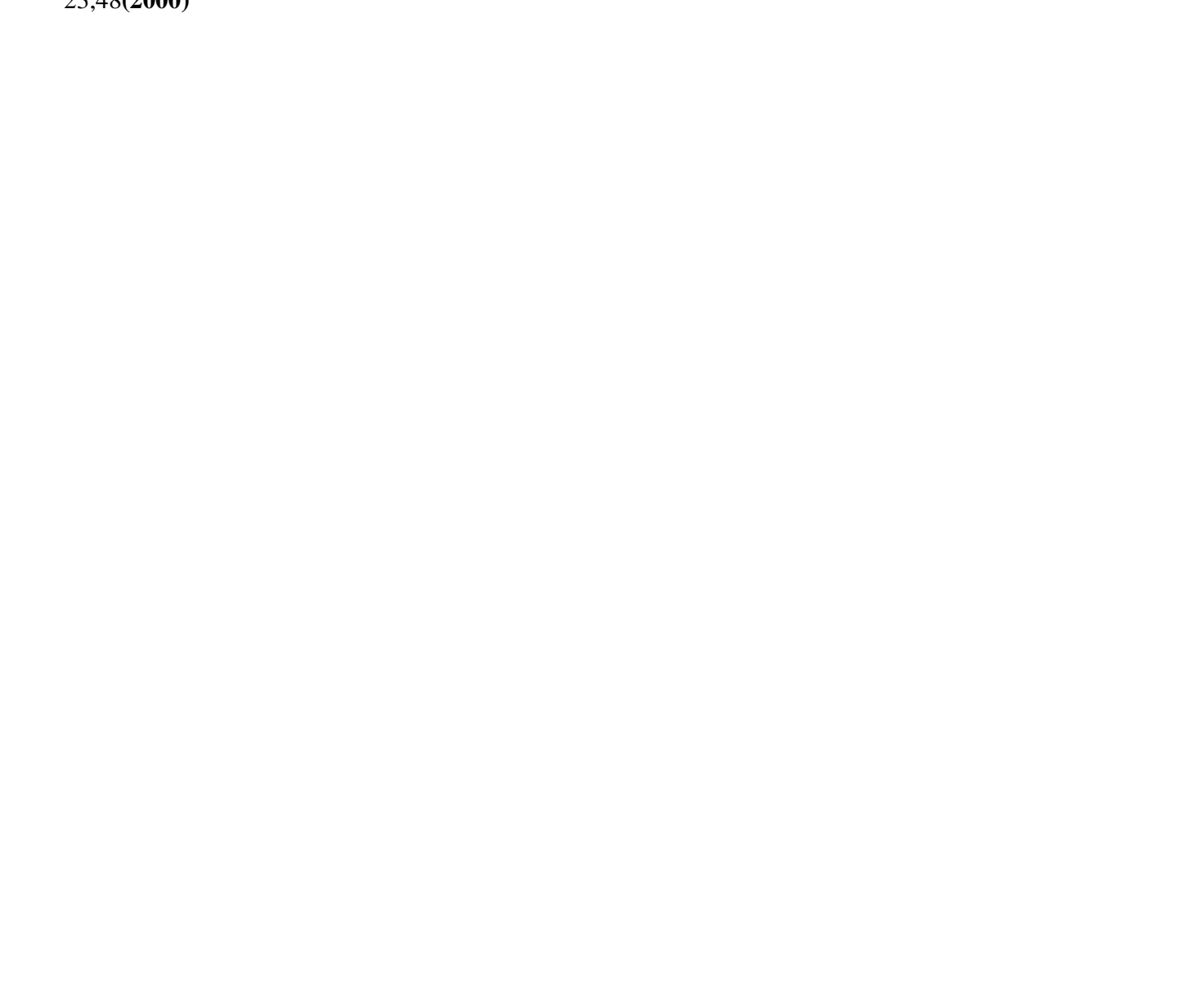

